\section{Tendência da mortalidade por neoplasias malignas selecionadas em Rio Branco, Acre, Brasil, 1980-2006}

\author{
Cancer mortality trends in Rio Branco, \\ Acre State, Brazil, 1980-2006
}

\author{
1 Programa de Mestrado em \\ Saúde Coletiva, Universidade \\ Federal do Acre, Rio Branco, \\ Brasil. \\ 2 Escola Nacional de Saúde \\ Pública Sergio Arouca, \\ Fundação Oswaldo Cruz, Rio \\ de Janeiro, Brasil. \\ Correspondência \\ S. Koifman \\ Escola Nacional de Saúde \\ Pública Sergio Arouca, \\ Fundação Oswaldo Cruz. \\ Rua Leopoldo Bulhões 1480, \\ Rio de Janeiro, RJ 21041-210, \\ Brasil. \\ koifman@ensp.fiocruz.br
}

\section{Abstract}

Time trends in cancer incidence and mortality in the Western Amazon remain unknown. This study explored age-standardized cancer mortality rates according to anatomical site in Rio Branco, Acre State, Brazil, by constructing linear regression time trend models. Cancer mortality showed an increasing but inconstant trend in men and stability in women. At the end of the time series, the highest cancer rates among women were for the cervix, lung, liver and intrahepatic biliary tract, stomach, and breast. Among men, the highest rates were for cancer of the lung, prostate, liver and intra-hepatic biliary tract, stomach, and esophagus. The study showed an increasing mortality time trend for cancer of the prostate, breast, and lung and declining mortality rates for cervical cancer in women, lung cancer in men, and stomach cancer in both sexes. The high mortality rate from liver cancer merits attention, considering the high hepatitis $B$ and $C$ infection rates in the State of Acre.

Neoplasms; Mortality; Temporal Distribution
Juliano de Pádua Nakashima 1

Sérgio Koifman 2

Rosalina Jorge Koifman 2

\section{Introdução}

As informações sobre a mortalidade têm sido a principal fonte para a compreensão do perfil epidemiológico das populações 1 . A taxa de mortalidade por câncer estima o risco de morte por neoplasias malignas e dimensiona a sua magnitude como problema de saúde pública ${ }^{2}$.

No Brasil, os dados sobre mortalidade são regularmente obtidos no Sistema de Informações sobre Mortalidade (SIM), sistema gerido pelo Departamento de Análise de Situação de Saúde, da Secretaria de Vigilância em Saúde, em conjunto com as secretarias estaduais e municipais de saúde. O documento oficial do SIM é a Declaração de Óbito (DO) 3 .

Na década de 1980, o câncer era a quinta causa de morte na população brasileira, sendo responsável por $10 \%$ dos óbitos no país 4 . A taxa de mortalidade correspondia a 117,5 óbitos por 100 mil habitantes no sexo masculino, e a 90,7 por 100 mil no sexo feminino ${ }^{5}$. Em 2000, as neoplasias malignas passaram a figurar como a terceira causa mais comum de óbito na população geral 4, com taxas de mortalidade de 235,8 por 100 mil homens e de 187,5 por $100 \mathrm{mil} \mathrm{mu-}$ lheres 5. Em 2007, o câncer tornou-se a segunda causa de óbito mais frequente, correspondendo a $15,4 \%$, do total de óbitos, atrás apenas das doenças do aparelho circulatório (29,4\% do total de óbitos) (Departamento de Informática do SUS. Indicadores de mortalidade: C.4 mortalidade 
proporcional por grupos de causas. http://tab net.datasus.gov.br/cgi/tabcgi.exe?idb2009/c04. def, acessado em 23/Fev/2010).

De acordo com o Instituto Nacional de Câncer (INCA. Atlas de mortalidade por câncer no Brasil. http://mortalidade.inca.gov.br/index.jsp, acessado em 23/Fev/2010), a distribuição das taxas de mortalidade por câncer ajustadas por idade, no período de 1980 a 2006, variou no sexo masculino entre os seguintes valores: 133,3/100 mil em Belém (Pará); 149,0/100 mil em São Paulo; 113,5/100 mil em Fortaleza (Ceará); 121,0/100 mil em Goiânia (Goiás); e de 189,1/100 mil em Porto Alegre (Rio Grande do Sul). No sexo feminino, as taxas foram de 94,1/100 mil em Belém; 96,5/100 mil em São Paulo; 83,5/100 mil em Fortaleza; 86,8/100 mil em Goiânia e de 113,0/100 mil em Porto Alegre.

Ainvestigação da tendência histórica de eventos relacionados à saúde, dentre eles a mortalidade por câncer, possibilita uma avaliação mais adequada das medidas de controle dos agravos à saúde. A distribuição temporal da incidência $\mathrm{e}$ mortalidade por câncer na Amazônia Ocidental é desconhecida, e o objetivo deste estudo foi analisar a tendência temporal da mortalidade por neoplasias malignas em localizações anatômicas selecionadas (mama, colo uterino, próstata, pulmão, estomago, cólon, reto, fígado e vias biliares) em residentes em Rio Branco (Acre).

\section{Material e métodos}

Foi realizado um estudo de séries temporais da mortalidade por neoplasias malignas na cidade de Rio Branco, durante o período de 1980 a 2006. Os dados de mortalidade foram obtidos no SIM do Departamento de Informática do SUS (DATASUS; http://www.datasus.gov.br), sendo selecionados todos os óbitos em residentes na cidade de Rio Branco ocorridos no período de 1980 a 2006 e cuja causa básica declarada incluiu a menção a neoplasias que apresentam as maiores taxas de mortalidade em Rio Branco: colo uterino, estômago, traqueia, brônquios e pulmão, próstata, mama, cólon, reto, fígado e vias biliares. O SIM utilizou a 9a revisão da Classificação Internacional de Doenças (CID-9) 6 para codificar os óbitos ocorridos de 1980 a 1995 e a 10a revisão (CID-10) 7, a partir de 1996.

Realizamos uma análise preliminar da mortalidade segundo idade e ano do óbito para todas as neoplasias malignas (exceto pele) e posteriormente para as localizações anatômicas com maiores taxa de mortalidade, a saber: colo uterino e porção não especificada (C53 e C55), estômago (C16), traqueia, brônquios e pulmão
(C33 e C34), próstata (C61), mama (C50), e fígado e vias biliares intra-hepáticas (C22).

As estimativas da população residente no município, segundo faixa etária tanto censitárias (1991, 1996, 2000) quanto intercensitárias (1979 a 2005) utilizadas como denominadores para os cálculos das taxas de mortalidade foram obtidas no DATASUS e fornecidas pelo Instituto Brasileiro de Geografia e Estatística (IBGE). As populações foram classificadas segundo sexo e faixa etária (menor que 20 anos, 20-29 anos, 30-39 anos, 40-49 anos, 50-59 anos, 60-69 anos e 70 anos ou mais) (DATASUS. Dados de população. http:// www.datasus.gov.br, acessado em 23/Fev/2010).

Inicialmente, foram calculadas as taxas brutas de mortalidade pela razão entre o número de óbitos por uma dada neoplasia maligna e a população estimada na data de 1o de julho em cada ano da série, sendo apresentadas por 100 mil habitantes. Em seguida, as taxas brutas de mortalidade foram padronizadas por idade pelo método direto, sendo utilizada como padrão a população mundial proposta por Segi 8 e modificada por Doll et al. 9 .

As taxas calculadas foram utilizadas na análise da tendência da mortalidade pelas diferentes neoplasias, através da estimativa de modelos de regressão. Para o processo de modelização, as taxas padronizadas de mortalidade, por cada localização de neoplasia (y) foram consideradas como variável dependente e, como variável independente (x), os anos do período de estudo.

$\mathrm{O}$ primeiro modelo testado foi o de regressão linear simples $\left(\mathrm{y}=\beta_{0}+\beta_{1} \mathrm{x}\right)$ e, a seguir, os modelos de segundo $\left(\mathrm{y}=\beta_{0}+\beta_{1} \mathrm{x}+\beta_{2} \mathrm{x}^{2}\right)$ e terceiro graus $\left(\mathrm{y}=\beta_{0}+\beta_{1} \mathrm{x}+\beta_{2} \mathrm{x}^{2}+\beta_{3} \mathrm{x}^{3}\right)$. Calculou-se o coeficiente de determinação $\left(\mathrm{R}^{2}\right)$, como uma medida da magnitude da variância da variável dependente analisada sendo explicada pela variância da variável preditora ano. Considerou-se como modelo mais adequado aquele apresentando maior significância estatística, sendo definido que, caso mais de um modelo se mostrasse semelhante sob o ponto de vista estatístico, seria escolhido o mais simples. Os modelos com valor de "p" igual ou menor a 0,05 foram considerados estatisticamente significativos.

Para se reduzir a correlação seriada entre os termos da equação de regressão, realizou-se a centralização da variável “ano" pelo ponto médio da série histórica. Portanto, para o período compreendido entre 1982 e 2004, o termo (X-1993) representa a variável centralizada. Para suavizar a série histórica, em função da oscilação dos pontos, proveniente do pequeno número de casos em determinados estratos, calculou-se a média móvel centrada em cinco termos. Nesse processo, o coeficiente alisado do ano i $\left(\mathrm{Y}_{\mathrm{ai}}\right)$ correspon- 
deu à média aritmética dos coeficientes dos dois anos anteriores (i-2), do próprio ano (i) e dos dois anos seguintes $(\mathrm{i}+2)$, correspondeu à fórmula:

$$
Y_{a i}=\frac{Y_{i-2}+Y_{i-1}+Y_{i}+Y_{i+1}+Y_{i+2}}{5}
$$

Utilizamos o programa Joinpoint, versão 3.4 (Statistical Research and Applications Branch, National Cancer Institute, Estados Unidos), fornecido pelo Instituto Nacional de Câncer Norte Americano para o cálculo da variação anual da mortalidade no período de 1980 a 2006 10. O programa executa uma regressão linear segmentada (jointpont regression) para estimar a variação anual percentual e identificar pontos em que há modificação da tendência. Foram ajustados sucessivamente modelos em que se assumiu um número diferente de "pontos" de modificação de tendência, desde 0 (caso em que a tendência é representada por um único segmento de reta) até 4. O modelo escolhido foi aquele com o maior número de pontos, em que se manteve a significância estatística $(\mathrm{p}<0,05)$.

A partir da inclinação estimada para cada segmento de reta (coeficiente de regressão) foi calculada a variação anual em porcentagem e sua significância estatística, estimada pelo método dos mínimos quadrados por um modelo linear generalizado, assumindo que as taxas seguem uma distribuição de Poisson.

O câncer de traqueia, brônquios e pulmão apresentou incompatibilidade entre a CID-9 e a CID-10. Essas três localizações eram codificadas na CID-9 como 162, ocorrendo na CID-10 sua separação em neoplasias da traqueia (C33) e em brônquios e pulmão (C34). Neste trabalho, optou-se por analisar conjuntamente as três localizações (C33 + C34).

Também foi encontrada incompatibilidade das CID em relação ao câncer de mama. Na
CID-9 a localização anatômica da mama era dividida nos códigos 174 e 175, câncer de mama no sexo feminino e masculino, respectivamente, enquanto que na CID-10, ambos os sexos foram incluídos sob o código C50. Como neste trabalho analisou somente câncer de mama no sexo feminino, a incompatibilidade não resultou em viés. A Tabela 1 apresenta a correspondência entre a CID-9 e a CID-10 segundo a localização anatômica primária do tumor (sítios selecionados no estudo).

Para cada segmento de reta, com inclinação estimada, foram calculados os limites do intervalo de 95\% de confiança (IC95\%).

\section{Resultados}

Os resultados da análise de tendência encontram-se na Tabela 2 e os resultados da análise da variação anual em porcentagem encontram-se na Tabela 3.

A taxa de mortalidade por câncer ajustada (todas as localizações anatômicas) no sexo masculino apresentou tendência de crescimento não constante (Figura 1). Entretanto, mais recentemente, entre 1996 e 2004, as taxas apresentaram crescimento constante, com variação anual percentual de 3,3. A taxa de mortalidade ajustada por idade aumentou de 75/100 mil para de 97,2/100 mil homens nesse período. No sexo feminino, a análise da tendência mostrou estabilidade das taxas no período estudado, e a taxa de mortalidade ajustada por idade foi de 82,2/ 100 mil mulheres em 2004 (Figura 2).

Em relação ao câncer de colo uterino, o modelo que melhor representou o comportamento da tendência foi o de segundo grau. A mortalidade pela neoplasia apresentou tendência decrescente e não constante. Entre os anos de 1994 e 2000,

\begin{tabular}{lcc}
\hline Sítio & CID-9 & CID-10 \\
\hline Traqueia, brônquios e pulmões & 162 & C33 e C34 \\
Estômago & 151 & C16 \\
Próstata & 185 & C61 \\
Mama & 174 & C50 \\
Colo uterino e porção não especificada & $179-180$ & C53 C C55 \\
Fígado e vias biliares intra-hepáticas & 155 & C22 \\
Esôfago & 150 & C15 \\
\hline
\end{tabular}


Resultados padronizados da análise de tendência das taxas de mortalidade por câncer (localizações selecionadas) segundo sexo. Rio Branco, Acre, Brasil, 1982 a 2004.

\begin{tabular}{|c|c|c|c|c|}
\hline Localização da neoplasia/Sexo & Modelo & $\mathrm{R}^{2}(\%)$ & Valor de $p\left(R^{2}\right)$ & Valor de $p(\beta)$ \\
\hline \multicolumn{5}{|l|}{ Todas as localizações } \\
\hline Feminino & $Y=70,32-0,95 x+0,06 x^{2}-0,008 x^{3}$ & 42 & NS & NS \\
\hline \multirow[t]{3}{*}{ Masculino } & $Y=86,52+2,02 x+0,1 x^{2}-0,017 x^{3}$ & 76 & $<0,001$ & $\beta 1<0,001$ \\
\hline & & & & $\beta^{2}=0,001$ \\
\hline & & & & $\beta^{3}<0,001$ \\
\hline \multicolumn{5}{|l|}{ Estômago } \\
\hline \multirow[t]{2}{*}{ Feminino } & $Y=6,0-0,14 x+0,016 x^{2}$ & 80 & $<0,001$ & $\beta^{3}=0,01$ \\
\hline & & & & $\beta 2<0,001$ \\
\hline Masculino & $Y=13,35-0,46 x$ & 49 & $<0,001$ & $\beta^{1}<0,001$ \\
\hline \multicolumn{5}{|l|}{ Pulmão } \\
\hline \multirow{3}{*}{ Feminino } & $Y=5,58+0,47 x+0,014 x^{2}-0,002 x^{3}$ & 88,7 & $<0,001$ & $\beta^{1}<0,001$ \\
\hline & & & & $\beta^{2}<0,01$ \\
\hline & & & & $\beta^{3}=0,01$ \\
\hline \multirow[t]{2}{*}{ Masculino } & $Y=12,55-0,52 x+0,005 x^{3}$ & 54,4 & $<0,001$ & $\beta 1<0,001$ \\
\hline & & & & $\beta 3<0,01$ \\
\hline \multicolumn{5}{|l|}{ Fígado } \\
\hline Masculino & $Y=11,2-0,056 x$ & 6,0 & NS & NS \\
\hline Feminino & $Y=5,5-0,1 x$ & 24 & $<0,001$ & $\beta 1=0,01$ \\
\hline \multicolumn{5}{|l|}{ Mama } \\
\hline \multirow[t]{2}{*}{ Feminino } & $Y=4,34+0,094 x+0,013 x^{2}$ & 56 & 0,003 & $\beta^{1}=0,001$ \\
\hline & & & & $\beta^{2}=0,003$ \\
\hline \multicolumn{5}{|l|}{ Esôfago } \\
\hline Masculino & $Y=2,8-0,05 x$ & 23 & $<0,001$ & $\beta 1=0,02$ \\
\hline \multirow[t]{2}{*}{ Colo uterino } & $Y=17,35-0,30 x-0,043 x^{2}$ & 64,7 & $<0,001$ & $\beta 1<0,001$ \\
\hline & & & & $\beta^{2}=0,001$ \\
\hline \multirow[t]{3}{*}{ Próstata } & $Y=8,97+0,37 x+0,016 x^{2}-0,002 x^{3}$ & 68,6 & 0,04 & $\beta^{1}<0,001$ \\
\hline & & & & $\beta 2=0,02$ \\
\hline & & & & $\beta^{3}=0,04$ \\
\hline
\end{tabular}

$\beta_{1}$ : valor do coeficiente $\beta_{1}$ na equação $Y=\beta_{0}+\beta_{1} x ; \beta_{2}$ : valor do coeficiente $\beta^{2}$ na equação $Y=\beta_{0}+\beta_{1} x+\beta_{2} x^{2} ; \beta_{3}$ : valor do coeficiente $\beta_{3}$ na equação $Y=\beta_{0}+\beta_{1} x+\beta_{2} x^{2}+\beta_{3} x^{3}$; NS: valor de $p$ estatisticamente não significativo.

observamos diminuição acentuada na taxa, com variação anual percentual negativa de 10,7. Após esse período, a taxa se estabilizou em aproximadamente 11 mortes por 100 mil mulheres.

Foi encontrada tendência decrescente, constante e estatisticamente significante (modelo linear) das taxas de mortalidade por câncer gástrico no sexo masculino. A taxa apresentou variação anual percentual negativa de $-3,4$. A taxa que correspondia a 19,5/100 mil em 1982 reduziu para 8,4/100 mil em 2004. No sexo feminino, constatamos tendência decrescente, porém não constante. A diminuição na taxa de mortalidade ocorreu particularmente no período entre 1982 e 1988 (variação anual percentual de -6) e entre 1995 e 1998 (variação anual percentual de -10,6), decaindo a taxa de 10,3/100 mil em 1982 para 6,5/100 mil em 2004.

O câncer de traqueia, brônquios e pulmão no sexo masculino apresentou, tendência decrescente, não constante, durante o período estudado. O modelo que melhor representou o comportamento da tendência da mortalidade pela neoplasia foi o de segunda ordem. As taxas declinaram de 17,2 óbitos por 100 mil em 1985 para 9,3/100 mil em 2002, com variação anual percentual negativa de 2,8. Após 2002, houve nova variação das taxas que chegaram em 2004 ao 


\section{Tabela 3}

Variação das taxas de mortalidade por câncer (localizações selecionadas) padronizada por idade (análise em jointpoint regression), segundo sexo. Rio Branco, Acre, Brasil, 1982 a 2004.

\begin{tabular}{|c|c|c|c|c|c|}
\hline CID-10 & Localização & Sexo & Período & $\begin{array}{c}\text { Variação anual } \\
\text { percentual }\end{array}$ & IC95\% \\
\hline \multirow[t]{10}{*}{ C00-C99 } & Todas & M & $1982-1985$ & 2,0 * & 0,$0 ; 4,0$ \\
\hline & & & 1985-1988 & $-4,0$ & $-8,1 ; 0,2$ \\
\hline & & & 1988-1993 & 0,7 & $-0,6 ; 2,0$ \\
\hline & & & 1993-1996 & $-7,9$ * & $-11,9 ;-3,7$ \\
\hline & & & $1996-2004$ & 3,3 * & 2,$9 ; 3,8$ \\
\hline & & $\mathrm{F}$ & $1982-1986$ & 1,1 & $-1,0 ; 3,3$ \\
\hline & & & 1986-1990 & $-5,0$ * & $-8,3 ;-1,6$ \\
\hline & & & 1990-1994 & $4,7 *$ & 1,$0 ; 8,5$ \\
\hline & & & 1994-1999 & $-4,7$ * & $-6.8 ;-2,5$ \\
\hline & & & 1999-2004 & 5,3 * & 3,$5 ; 7,1$ \\
\hline \multirow[t]{4}{*}{ C53 e C55 } & Colo uterino & $\mathrm{F}$ & $1982-1988$ & $-2,3$ & $-4,8 ; 0,3$ \\
\hline & & & 1988-1994 & 5,5 * & 2,$2 ; 9,0$ \\
\hline & & & $1994-2000$ & $-10,7$ * & $-13,9 ;-7,4$ \\
\hline & & & $2000-2004$ & 0,6 & $-6,0 ; 7,6$ \\
\hline \multirow[t]{9}{*}{ C16 } & Estômago & M & 1982-1984 & 1,0 & $-25,4 ; 36,7$ \\
\hline & & & 1984-1987 & $-20,8$ & $-44,5 ; 13,2$ \\
\hline & & & 1987-1992 & 15,5 * & 5,$7 ; 26,2$ \\
\hline & & & 1992-1998 & $-14,5$ * & $-19,8 ;-8,8$ \\
\hline & & & $1998-2004$ & 4,1 & $-2,4 ; 11,0$ \\
\hline & & $\mathrm{F}$ & $1982-1988$ & $-6,0$ * & $-8,3 ;-3,8$ \\
\hline & & & 1988-1995 & $-0,2$ & $-2,9 ; 2,6$ \\
\hline & & & 1995-1998 & $-10,6$ & $-25,6 ; 7,2$ \\
\hline & & & $1998-2004$ & $6,9 *$ & 3,$6 ; 10,3$ \\
\hline \multirow[t]{7}{*}{$\mathrm{C} 33$ e C34 } & Traqueia, brônquios e & M & $1982-1985$ & 14,8 * & 1,$7 ; 29,5$ \\
\hline & pulmão & & $1985-2002$ & $-2,8$ * & $-3,7 ;-1,9$ \\
\hline & & & $2002-2004$ & 16,1 & $-12,6 ; 54,0$ \\
\hline & & $\mathrm{F}$ & $1982-1984$ & 13,6 & $-16,4 ; 54,4$ \\
\hline & & & 1984-1989 & $-9,0$ * & $-16,7 ;-0,6$ \\
\hline & & & 1989-1996 & 14,0 * & 10,$0 ; 18,1$ \\
\hline & & & $1996-2004$ & $2,1 *$ & 0,$2 ; 4,1$ \\
\hline \multirow[t]{2}{*}{ C61 } & Próstata & M & 1982-1988 & $-3,6$ & $-9,8 ; 2,9$ \\
\hline & & & $1988-2004$ & 3,3 * & 1,$9 ; 4,7$ \\
\hline \multirow[t]{2}{*}{ C50 } & Mama & $\mathrm{F}$ & $1982-1993$ & $-2,0$ & $-4,7 ; 0,7$ \\
\hline & & & 1993-2004 & $5,5^{*}$ & 2,$9 ; 8,2$ \\
\hline \multirow[t]{7}{*}{$\mathrm{C} 22$} & Fígado e vias biliares & M & 1982-1991 & 4,1 * & 2,$1 ; 6,1$ \\
\hline & intra-hepáticas & & $1991-1996$ & $-8,5$ * & $-14,5 ;-2,1$ \\
\hline & & & $1996-2002$ & 7,9 * & 2,$8 ; 13,3$ \\
\hline & & & $2002-2004$ & $-8,5$ & $-24,2 ; 10,4$ \\
\hline & & $\mathrm{F}$ & $1982-1987$ & 10,1 * & 3,$3 ; 17,4$ \\
\hline & & & $1987-2000$ & $-6,3$ * & $-8,0 ;-4,6$ \\
\hline & & & $2000-2004$ & 17,7 * & 5,$5 ; 31,3$ \\
\hline
\end{tabular}

CID-10: 10ạ revisão da Classificação Internacional de Doenças; IC95\%: intervalo de 95\% de confiança; F: feminino;

M: masculino.

* Significativamente diferente de 0 (valor de $p<0,05$ ). 
Figura 1

Taxa de mortalidade por câncer (todas as localizações) padronizada por idade, sexo masculino. Rio Branco, Acre, Brasil, 1982 a 2004.

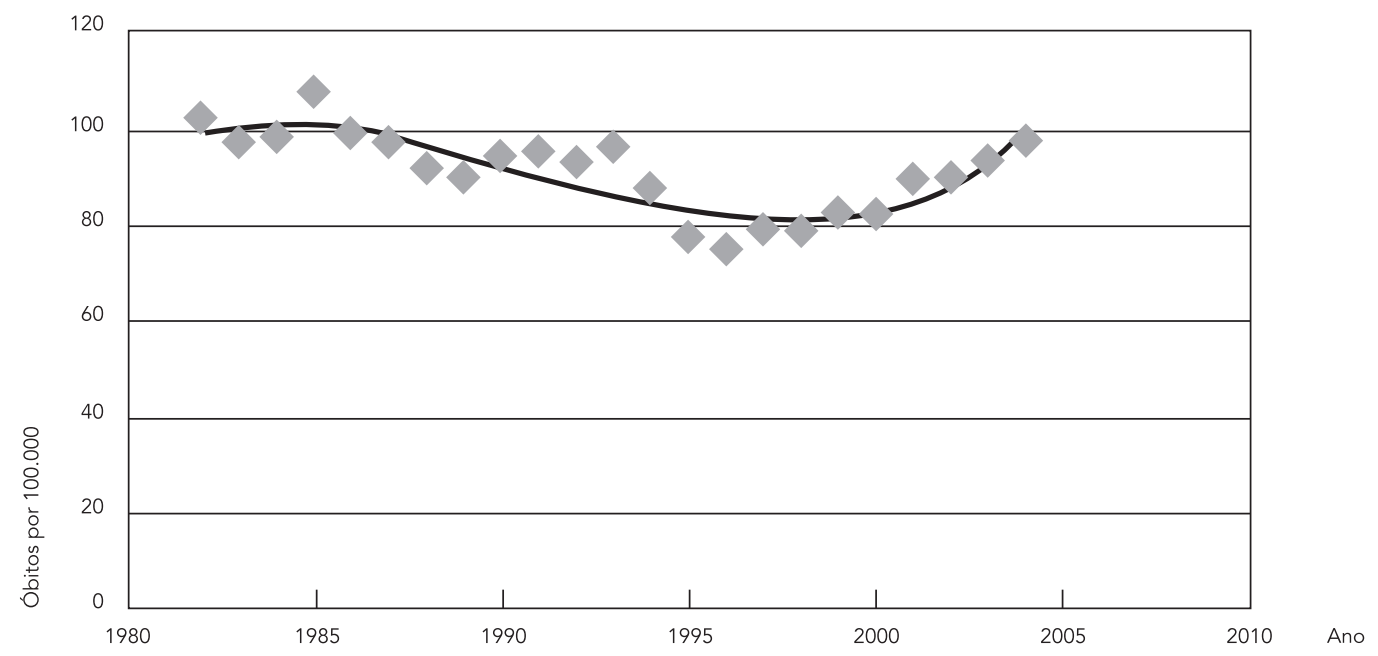

Figura 2

Taxa de mortalidade por câncer (todas as localizações) padronizada por idade, sexo feminino. Rio Branco, Acre, Brasil, 1982 a 2004.

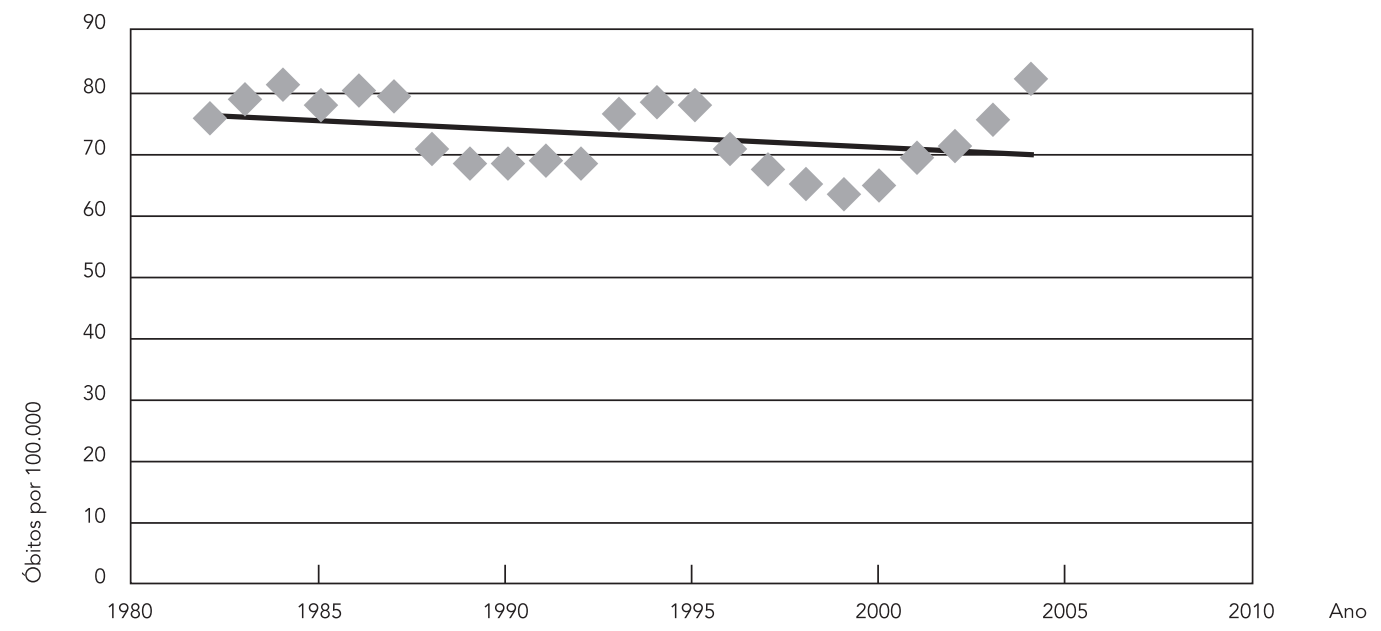


valor de 12,6/100 mil. No sexo feminino, as taxas mantiveram-se relativamente estáveis durante o período entre 1982 e 1984, oscilando entre 3,7 óbitos por 100 mil e 4,9/100 mil. Entretanto, nos anos mais recentes, verificamos crescimento significativo da taxa que em 1989 correspondia a 3,2 óbitos por 100 mil e elevou-se para 7,6/100 mil em 1996 (variação anual percentual de 14). Após tal período, a taxa manteve tendência de crescimento, contudo menos acentuada (variação anual percentual de 2,1), atingindo em 2004 o valor de 10,4/100 mil.

A taxa padronizada de mortalidade por câncer de próstata apresentou aumento significativo no período estudado, mas não constante. Foi observada grande oscilação nas taxas durante o período estudado, sendo a tendência de crescimento evidente após 1988, ano a partir do qual a taxa apresentou variação anual percentual de 3,3, atingindo em 2004 o valor de 12,1 óbitos por 100 mil homens.

Ocorreu tendência a aumento estatisticamente significativo nas taxas padronizadas de mortalidade por câncer da mama. A tendência, todavia, não foi constante, e o modelo que melhor representou seu comportamento foi o de segundo grau. Após o ano de 1993, a taxa que apresentava valor de 2,9/100 mil revelou crescimento acentuado com variação anual percentual de 5,5, chegando em 2004 a 6,4 óbitos por $100 \mathrm{mil}$ mulheres.

As taxas de mortalidade por neoplasia maligna de fígado e vias biliares intra-hepáticas tanto no sexo masculino, quanto no sexo feminino, apresentaram grande flutuação no período estudado, sugerindo estabilidade das distribuições. As taxas oscilaram de 8,8 a 14,1/100 mil no sexo masculino e de 3,6 e 8,4 no sexo feminino. Nos anos mais recentes, período entre 2002 e 2006, as taxas foram de 11,7 no sexo masculino e de $6,7 / 100$ mil no sexo feminino.

As taxas padronizadas de mortalidade por câncer do esôfago permaneceram estáveis durante o período estudado no sexo masculino. Foi observado aumento acentuado das taxas no período de 1982 a 1987. Após esse período, até o ano de 1997, as taxas declinaram menos intensamente, atingindo patamares apresentados no início da série. Mais recentemente, no período entre 2002 e 2006, a mortalidade pela neoplasia apresentou taxa padronizada de 1,9/100 mil.

\section{Discussão}

Um aspecto relevante a ser considerado em estudos de tendência da mortalidade, como aqui apresentado em relação às neoplasias, diz res- peito à qualidade dos dados de mortalidade ao longo do tempo. Cabe destacar que de acordo com as informações disponibilizadas pelo DATASUS, a distribuição de causas mal definidas de óbito em Rio Branco manteve-se estável e em um patamar elevado durante todo o período de estudo: $21 \%$ em 1980, $26,6 \%$ em 1985 , 24,1\% em 1990, 21,8\% em 1995, e 22,4\% em 2000 e 2004. Dessa maneira, as conclusões em estudos de séries temporais de mortalidade naquele município no período aqui analisado devem ser tomadas com cautela, em virtude das limitações inerentes na consistência dos dados de mortalidade.

Uma vez ressaltadas tais considerações, foi possível observar no presente estudo que a mortalidade por câncer (todas as localizações anatômicas) em Rio Branco apresentou tendência de crescimento não constante no sexo masculino e de estabilidade no sexo feminino. Um estudo que avaliou a tendência temporal da mortalidade por câncer no período compreendido entre 1980 e 1995, mostrou que as taxas de mortalidade apresentaram variação percentual de $-0,3 \%$ nos homens, diminuindo de 62,5/100 mil para $62,3 / 100$ mil e de $-4,8 \%$ nas mulheres, reduzindo de 52,5/100 mil para 50,1/100 mil 11. Desde a década de 1970, uma queda constante e sistemática da mortalidade por câncer do colo do útero vem sendo encontrada na maioria dos países latino-americanos, especialmente em mulheres de meia idade ${ }^{12}$. No Brasil, no período de 1981 a 2006, somente a Região Sul mostrou aumento no risco de morte por câncer do colo do útero. As demais regiões do país apresentaram tendência de estabilização ou de queda no risco de morte pela neoplasia 4 .

Algumas hipóteses podem ser levantadas para a tendência decrescente observada na mortalidade por câncer de colo uterino em Rio Branco. A redução poderia estar refletindo modificações decorrentes da melhoria no acesso aos serviços de saúde, indicando adesão aos programas de prevenção, e de que o diagnóstico precoce de lesões intraepiteliais cervicais e seu tratamento têm sido mais efetivos.

A queda da mortalidade por câncer de estômago é um fenômeno verificado mundialmente. A queda pode ser parcialmente decorrente de uma diminuição na incidência, uma vez que o tratamento específico não evoluiu significativamente nos últimos anos 13, e também da modificação de hábitos alimentares, como o maior consumo de frutas e verduras frescas decorrente da disponibilidade de geladeiras. O câncer gástrico apresentou no presente estudo tendência de decréscimo em Rio Branco, em ambos os sexos. A tendência de declínio observada provavelmen- 
te permanecerá por mais alguns anos (modelos linear no sexo masculino e de segundo grau no feminino) e talvez decorra de alterações na exposição aos fatores de risco, como por exemplo, a introdução da refrigeração elétrica e outras formas de conservação dos alimentos, o melhoramento do saneamento básico e a modificação no hábito alimentar (aumento da ingestão de frutas, legumes e verduras, redução do uso do sal) 14 . A Região Norte também apresentou tendência de decréscimo. No Estado do Pará, no período de 1980 a 1997, foi verificada diminuição da tendência em ambos os sexos, porém mais acentuada nos homens 15 .

A Região Norte do país vem apresentando, desde 1979, tendência de aumento na mortalidade por câncer de pulmão em ambos os sexos, entretanto é mais acentuada no sexo feminino 16 . Os achados do presente estudo indicam que Rio Branco apresenta tendência de decréscimo no sexo masculino e de crescimento no sexo feminino. Esse achado é compatível com a literatura nacional 15 e internacional $17,18,19$ e pode ser explicado pela associação entre o câncer de pulmão e a prevalência do tabagismo 20. Como o tabagismo é o principal fator de risco para o câncer de pulmão 21 , a tendência atual da mortalidade pela neoplasia reflete o hábito de fumar das gerações passadas 22 . Assim, a tendência decrescente observada no sexo masculino reflete provavelmente um declínio no tabagismo ocorrido há pelo menos 20/30 anos, e a tendência aumento na taxa de mortalidade no sexo feminino seria o resultado do aumento do tabagismo ocorrido entre as mulheres nas décadas de 1960 e 1970. Adicionalmente, alguns fatores ocupacionais, ambientais e dietéticos também podem ter exercido alguma influência sobre a tendência favorável nos homens 23 .

O câncer de próstata apresentou tendência de crescimento não constante em Rio Branco. Também foi encontrada tendência de crescimento na mortalidade pela neoplasia na Região Norte. A taxa de mortalidade pela neoplasia registrou aumento de 3,1/100 mil em 1980 para 6,4/100 mil em 1995 11. Da mesma forma, também se verificou aumento das taxas de mortalidade por câncer de próstata no Estado de São Paulo, no período de 1970 a 1992 24, e no Rio Grande do Sul, no período de 1979 a 1995 25. Como hipótese explicativa para esses resultados, é importante destacar que a expansão e a melhoria das técnicas de diagnóstico precoce, como o rastreamento pela dosagem sérica do antígeno prostático específico (PSA), podem ter conduzido a uma maior precocidade na detecção desta neoplasia maligna, que outrora poderia permanecer não diagnosticada 26. Além disso, é importante con- siderar que a maior longevidade e controle das demais doenças crônico-degenerativas, como a hipertensão e o diabetes, por exemplo, contribuem para a maior exposição ao risco de desenvolver e morrer por câncer de próstata.

Foi observada tendência de aumento nas taxas de mortalidade por câncer de mama em Rio Branco. Na Região Norte também se encontrou tendência de aumento da mortalidade pela neoplasia. No período entre 1980 e 1995, houve variação de $37,5 \%$ na taxa padronizada de mortalidade pela neoplasia que aumentou de 3,2 óbitos por 100 mil mulheres para 4,4/100 mil mulheres 11. No Estado de São Paulo, no período de 1970 a 1992, foi observado aumento de $19,7 \%$ nos coeficientes de mortalidade pela neoplasia, salientando que esta era a principal causa de morte por câncer em mulheres paulistas 25 . Na Região Sul do país, no período de 1980 a 2002, observou-se crescimento das taxas de mortalidade por câncer de mama nos três estados da região 27 . O aumento verificado em Rio Branco pode estar relacionado ao envelhecimento populacional. A exposição a substâncias químicas, entre as quais o uso de hormônios exógenos, pesticidas, entre outros, bem como o aumento da prevalência da obesidade 28 e fatores dietéticos 29 , podem também ter desempenhado um papel na recente tendência ascendente da mortalidade por câncer de mama. A ausência de um programa de rastreamento que atinja toda a população feminina suscetível, associada à precária assistência terapêutica prestada, provavelmente contribui para que a tendência na mortalidade permaneça crescente por alguns anos.

O câncer de fígado e vias biliares intra-hepáticas representou, em 2004, a terceira causa mais frequente de óbito por câncer em ambos os sexos. A infecção pelos vírus da hepatite B e C, um dos fatores etiológicos mais importantes para o hepatocarcinoma 30 , é endêmica na região 31 . Segundo dados do Ministério da Saúde, em 2005, as taxas de mortalidade por hepatites virais $\mathrm{B}$ e C no Acre foram as maiores do Brasil 32. Esse achado pode explicar a magnitude das taxas de mortalidade pela neoplasia no município. Entretanto, o registro de óbitos por câncer metastático para o fígado e notificados como o câncer primário do órgão, também justificaria a observação de taxas de mortalidade tão elevadas.

Os resultados apresentados neste estudo apresentam algumas limitações a serem destacadas. Inicialmente, deve-se tomar em consideração que a atenção prestada aos pacientes com câncer durante o período de estudo deste trabalho era majoritariamente realizada fora do Estado do Acre. O Hospital de Câncer de Rio Branco iniciou suas atividades em 2007, e até então 
a maioria dos pacientes era encaminhada para tratamento em outros estados. Assim, embora a informação sobre o município de residência seja disponibilizada nos dados de mortalidade do DATASUS, é possível supor que as informações sobre a casuística de óbitos, e consequentemente as taxas de mortalidade por câncer na série analisada nesta investigação, possam estar subestimadas ao longo de todo o período analisado.

Por outro lado, a investigação apresenta alguns pontos fortes. Trata-se do primeiro estudo realizado de forma abrangente que avalia a magnitude das tendências da mortalidade por câncer em Rio Branco, e dessa maneira, retrata uma parcela do quadro epidemiológico da mortalidade por câncer na Amazônia Ocidental.

Por tal razão, considerando-se a inexistência de registros de câncer de base populacional em toda aquela região, as tendências descritas do padrão de mortalidade por câncer neste trabalho poderão vir a contribuir para a discussão do quadro epidemiológico regional atual das neoplasias e o estabelecimento de políticas públicas para seu diagnóstico precoce e controle.

\section{Resumo}

A distribuição temporal da incidência e mortalidade por câncer na Amazônia Ocidental é desconhecida. As tendências das taxas de mortalidade por câncer ajustadas por idade nas localizações anatômicas apresentando maior magnitude entre 1980-2006 em Rio Branco, Acre, Brasil, foram modelizadas por meio de regressão linear. A tendência temporal da mortalidade por câncer em homens revelou padrão de crescimento não constante, e estabilidade em mulheres. Ao final da série, as taxas mais elevadas em mulheres foram, em ordem decrescente, colo uterino, pulmão, fígado e vias biliares intra-hepáticas, estômago e mama. No sexo masculino, foram pulmão, próstata, fígado e vias biliares intra-hepáticas, estômago e esôfago. O padrão observado revela elevação na mortalidade por câncer de mama e pulmão em mulheres, declínio de câncer de colo uterino, pulmão em homens, e estômago em ambos os sexos. A elevada mortalidade por câncer de figado merece destaque em decorrência das elevadas taxas de infecção pelo vírus da hepatite B e C no Acre.

\section{Conclusões}

As informações da mortalidade por câncer analisadas na população residente de Rio Branco, são sugestivas de que seu padrão de distribuição durante o período 1980 a 2006 guarda, em seu conjunto, características similares com as tendências descritas nas populações das demais regiões do país. Entre estas, cabe destacar as tendências observadas quanto à elevação na mortalidade por câncer de próstata, de câncer de mama e de pulmão em mulheres, bem como o declínio na mortalidade pelas neoplasias de colo uterino, de câncer de pulmão em homens, e de câncer de estômago em ambos os sexos. Por outro lado, e de forma distinta do observado em outras regiões, foi constatada uma alta mortalidade por câncer de fígado e vias biliares intra-hepáticas, representando a terceira maior causa de morte por câncer em ambos os sexos.

\section{Colaboradores}

J. P. Nakashima, S. Koifman e R. J. Koifman participaram da concepção, redação, revisão e aprovação final do estudo.

\section{Agradecimentos}

A realização desta investigação foi parcialmente apoiada pela Coordenação de Aperfeiçoamento de Pessoal de Nível Superior (CAPES) através da Associação Temporária estabelecida entre a Universidade Federal do Acre (Mestrado em Saúde Coletiva) e a Escola Nacional de Saúde Pública Sergio Arouca, Fundação Oswaldo Cruz (Programa de Pós-graduação em Saúde Pública e Meio Ambiente). R. J. Koifman e S. Koifman desenvolvem atividades de pesquisa apoiadas pelo Conselho Nacional de Desenvolvimento Científico e Tecnológico (CNPq) e Fundação de Amparo à Pesquisa do Estado do Rio de Janeiro (FAPERJ). 


\section{Referências}

1. Lagiou P, Adami HO, Trichopoulos D. Measures and estimates of cancer burden. In: Adami H, Hunter D, Trichopoulos D, editors. Textbook of cancer epidemiology. Oxford: Oxford University Press; 2008. p. $43-4$.

2. Rede Interagencial de Informação para a Saúde. Indicadores básicos para a saúde no Brasil: conceitos e aplicações. 2a Ed. Brasília: Organização PanAmericana da Saúde; 2008.

3. Fundação Nacional de Saúde. Manual de instruções para o preenchimento da declaração de óbito. 3a Ed. Brasília: Fundação Nacional de Saúde; 2001.

4. Secretaria de Vigilância em Saúde, Ministério da Saúde. Saúde Brasil 2004: uma análise da situação de saúde. Brasília: Ministério da Saúde; 2004.

5. Cervi A, Hermsdorff HHM, Ribeiro RCL. Tendência da mortalidade por doenças neoplásicas em 10 capitais brasileiras, de 1980 a 2000. Rev Bras Epidemiol 2007; 8:407-18.

6. Organização Mundial da Saúde. Manual da classificação estatística internacional de doenças, lesões e causas de óbito. 9a Ed. São Paulo: Centro da OMS para Classificação de Doenças em Português; 1975.

7. Organização Mundial da Saúde. Classificação estatística internacional de doenças e problemas relacionados à saúde. 10th Ed. São Paulo: Centro Colaborador da Organização Mundial da saúde para a Classificação de Doenças em Português; 1995.

8. Segi M. Cancer mortality for selected sites in 24 countries (1950-57). Sendai: Department of Public Health, Tohoku University School of Medicine; 1960.

9. Doll R, Payne P, Waterhouse JAH, editors. Cancer incidence in five continents. v. I. Geneva: Union Internationale Contre le Cancer; 1966.

10. Kim HJ, Fay MP, Feuer EJ, Midthune DN. Permutation tests for joinpoint regression with applications to cancer rates. Stat Med 2000; 19:335-51.

11. Wünsch Filho V, Moncau JE. Mortalidade por câncer no Brasil 1980-1995: padrões regionais e tendências temporais. Rev Assoc Med Bras 2002; 48:250-7.

12. Bocciolone L, La Vecchia C, Levi F, Lucchini F, Franceschi S. Trends in uterine cancer mortality in the Americas, 1955-1988. Gynecol Oncol 1993; 51:335-44.

13. Doll R. Are we winning the fight against cancer? An epidemiological assessment. EACR - Mühlbock memorial lecture. Eur J Cancer 1990; 26:500-8.

14. Plummer M, Franceschi S, Muñoz N. Epidemiology of gastric cancer. IARC Sci Publ 2004; (157):311-26.

15. Resende ALS, Mattos IE, Koifman S. Mortalidade por câncer gástrico no estado do Pará, 1980-1997. Arq Gastroenterol 2006; 43:247-52.

16. Boing AF, Rossi TF. Tendência temporal e distribuição espacial da mortalidade por câncer de pulmão no Brasil entre 1979 e 2004: magnitude, padrões regionais e diferenças entre sexos. J Bras Pneumol 2007; 33:544-51.
17. Jemal A, Chu KC, Tarone RE. Recent trends in lung cancer mortality in the United States. J Natl Cancer Inst 2001; 93:277-83.

18. Parkin DM, Bray F, Ferlay J, Pisani P. Global cancer statistics, 2002. CA Cancer J Clin 2005; 55:74-108.

19. Bray F, Tyczynski JE, Parkin DM. Going up or coming down? The changing phases of the lung cancer epidemic from 1967 to 1999 in the 15 European Union countries. Eur J Cancer 2004; 40:96-125.

20. Doll R, Hill AB. Mortality in relation to smoking: ten years' observations of British doctors. BMJ 1964; 1:1460-7.

21. Parkin DM. Epidemiology of cancer: global patterns and trends. Toxicol Lett 1998; 102/103:227-34.

22. World Health Organization. Tobacco or health: a global status report. Geneva: World Health Organization; 1997.

23. Blot WJ, Fraumeni Jr. JF. Cancer of the lung and pleura. In: Schottenfeld D, Fraumeni Jr. JF, editors. Cancer epidemiology and prevention. New York: Oxford University Press; 1996. p. 637-65.

24. Fonseca LAM, Mameri CP. Mortalidade por câncer no Estado de São Paulo. Oncol Atual 1992; 2:6-14.

25. Hallal AL, Gotlieb SLD, Latorre MRDO. Evolução da mortalidade por neoplasias malignas no Rio Grande do Sul, 1979-1995. Rev Bras Epidemiol 2001; 4:168-77.

26. Sun L, Caire AA, Robertson CN, George DJ, Polascik TJ, Maloney KE, et al. Men older than 70 years have higher risk prostate cancer and poorer survival in the early and late prostate specific antigen eras. J Urol 2009; 182:2242-8.

27. Gonçalves ATC, Jobim PFC, Vanacor R, Nunes LN, Albuquerque IM, Bozzetti MC. Câncer de mama: mortalidade crescente na Região Sul do Brasil entre 1980 e 2002. Cad Saúde Pública 2007; 23:1785-90.

28. Filozof C, Gonzalez C, Sereday M, Mazza C, Braguinsky J. Obesity prevalence and trends in LatinAmerican countries. Obes Rev 2001; 2:99-106.

29. Cibeira GH, Guaragna RM. Lipídio: fator de risco e prevenção do câncer de mama. Rev Nutr 2006; 19:65-75.

30. Hepatitis viruses. IARC Monogr Eval Carcinog Risks Hum 1994; 59:1-255.

31. Viana S, Paraná R, Moreira RC, Compri AP, Macedo V. High prevalence of hepatitis B virus and hepatitis D virus in the western Brazilian Amazon. Am J Trop Med Hyg 2005; 73:808-14.

32. Secretaria de Vigilância em Saúde, Ministério da Saúde. Sistema nacional de vigilância em saúde: relatório de situação - Acre. 2a Ed. Brasília: Ministério da Saúde, 2007.

Recebido em 18/Nov/2010

Versão final reapresentada em 20/Mar/2011 Aprovado em 07/Abr/2011 